\title{
La realización de feminidad entre jóvenes veinteañeras: el papel de las confidencias sobre las relaciones con los hombres en la conversación juvenil femenina
}

The realisation of femininity among women in their twenties: the role of reciprocal self-disclosure concerning relationships with men in feminine juvenile conversation

\author{
Virginia Acuña Ferreira
}

Universidad de Vigo. Correo electrónico: californiana1@hotmail.es

Este artículo aborda la construcción de identidades femeninas en la conversación natural, apoyándose en las teorías constructivistas/performativas del género y en el concepto de Comunidad de Práctica, de acuerdo con las tendencias actuales en los estudios sobre Lengua, Género y Sexualidad. En primer lugar, se revisa la trayectoria de este campo, explicando sus inicios con la búsqueda de "diferencias de género" en la comunicación, las razones del cambio a este marco constructivista/performativo y las bases de esta nueva perspectiva, así como las críticas sobre su aplicación que se han planteado más recientemente desde el Análisis Conversacional (AC). En segundo lugar, el artículo se centra en el análisis de extractos de una conversación natural entre tres jóvenes veinteañeras que comparten confidencias sobre sus relaciones heterosexuales. El análisis demuestra las importantes funciones sociales e interpersonales que desempeña este acontecimiento comunicativo, actuando como una forma de realizar amistad y de desplegar identidades juveniles impregnadas de feminidad. Por una parte, la conversación está marcada por la recreación de un estilo de interacción estereotípicamente femenino, centrado en la creación de intimidad, en la expresión de solidaridad y en el refuerzo de la "conexión" entre las participantes. Por otra parte, las relaciones con los hombres son abordadas desde el punto de vista de los sentimientos y de la formación de pareja, a través de la producción de numerosas historias que aportan detalles sobre las experiencias personales de las participantes en este sentido y, especialmente, sobre la forma en que está evolucionando la "relación informal" que mantiene una de ellas.

Palabras clave: identidades femeninas, conversación natural, jóvenes veinteañeras, confidencias, relaciones con los hombres.

This paper analyzes the construction of feminine identities in natural conversation, drawing on constructionist/performative theories of gender and the concept of Community of Practice, in concordance with current trends in studies on Language, Gender and Sexuality. First, the state of the art in this field is reviewed, explaining its beginnings in the pursuit of "gender 
differences" in communication, the reasons for change to a constructionist/performative framework and the basis of this new perspective, as well as the criticisms of its application that have been more recently raised from Conversation Analysis (CA). Second, the paper focuses on the analysis of excerpts taken from a natural conversation among three twenties women who engage in reciprocal self-disclosure about their relationships with men. The analysis demonstrates the important social and interpersonal functions played by this communicative event, acting as a way for doing friendship and displaying youth identities which emerge as imbued with femininity. On the one hand, the conversation is marked by the performance of a stereotypically feminine style of interaction, focusing on the creation of intimacy, the expression of solidarity and the strengthening of "rapport" between the participants. On the other hand, relationships with men are approached from the point of view of feelings and matching, through the telling of a great number of stories that provide details on the personal experiences of the participants in this respect and, especially, on how it is evolving the "informal relationship" which one of the girls is maintaining.

Keywords: feminine identities, natural conversation, twenties women, reciprocal selfdisclosure, relationships with men.

\section{LAS DIFERENCIAS DE GÉNERO EN LA INTERACCIÓN}

El análisis de diferencias en las formas de hablar y de interactuar de las mujeres y los hombres ha sido uno de los principales objetivos de los estudios sobre Lengua, Género y Sexualidad desde sus inicios. Este campo comenzó a formarse a partir de los años setenta en los EE.UU y en el Reino Unido y su desarrollo en la actualidad continúa estando especialmente ligado al mundo anglófono. Las primeras investigaciones estuvieron muy influenciadas por la perspectiva feminista de trabajos pioneros como los de Lakoff $(1975,2004)$ o Thorne y Henley (1975) y se centraron en analizar cómo los hombres ejercen poder en la interacción con mujeres, por ejemplo a través de las interrupciones (Zimmermann y West 1975) o de la falta de apoyo a los temas propuestos por sus interlocutoras (Fishman 1980, 1983). Se trata de estudios que argumentan sobre la dominación masculina de las interacciones mixtas, por lo que suelen agruparse dentro de la llamada "aproximación de la dominación". En contraste, la "teoría de las dos culturas" de Maltz y Borker $(1982,1995)$ propuso considerar este tipo de situaciones comunicativas como un encuentro intercultural en el que son frecuentes los malentendidos, debido a que las mujeres y los hombres tienen estilos comunicativos diferentes, que aprenden desde la infancia, cuando juegan e interactúan principalmente con miembros de su mismo sexo.

Deborah Tannen $(1990,1991)$ aplicó esta última perspectiva en su libro You just don't understand, donde analiza numerosos casos de problemas comunicativos dentro de la pareja como malentendidos interculturales que se deben a esas diferencias de estilo comunicativo. Su trabajo generó una gran controversia dentro del campo, ya que algunas autoras criticaron la falta de atención a las asimetrías de poder entre hombres y mujeres en la explicación de muchos de los ejemplos analizados (véase, por ejemplo, Henley y Kramarae 1991; Trömel-Plötz 1991; Talbot 2010; véase la respuesta a estas críticas en Tannen 1994, 1996). Frente a la aproximación de la dominación, este enfoque intercultural dio lugar al surgimiento y expansión de 
una "aproximación de la diferencia", en la que suele incluirse a todas aquellas investigaciones que argumentan sobre la existencia de diferencias de género en las formas de hablar y de comunicarse, sin hacer alusión a cuestiones de poder o dominación (véase Acuña Ferreira 2009a, 2011; Talbot 2010).

Una característica muy notable de las investigaciones en este campo, común a las dos aproximaciones comentadas, es que se basaron generalmente en el análisis cuantitativo de numerosos rasgos cuantificables de la interacción, como las interrupciones, las señales de retroalimentación o respuestas mínimas, las tag questions o preguntas coletilla y otras formas de atenuación de las afirmaciones, como creo o supongo ${ }^{1}$ (véase Aries 1996). No obstante, también tuvieron mucha repercusión las investigaciones cualitativas de Jennifer Coates (1988, 1996, 1997a, 1997b, 2013), basadas en conversaciones de su propio grupo de amigas británicas, en las que se resalta la importancia de la comunicación como forma de "hacer amistad" entre las mujeres. Esta autora argumenta que en los grupos femeninos lo más importante son las relaciones interpersonales y la construcción de intimidad y solidaridad, por lo que los temas de conversación suelen elaborarse conjuntamente, de acuerdo con las normas de un turno colaborativo (Edelsky 1981). Apoyándose en datos de conversación amistosa masculina, Coates (1997c, 2013) sostiene que entre los hombres, en cambio, lo más importante es el intercambio de información y la reafirmación del estatus personal dentro del grupo; por ello, afirma que la conversación masculina tiende a centrarse en debates sobre cuestiones impersonales en los que se compite por adjudicarse el estatus de "experto" y se prefiere un turno individual, que se orienta a las normas de "uno a uno" establecidas por Sacks, Schegloff y Jefferson (1974), evitando el habla simultánea (véase también Edelsky 1981).

Tannen $(1990,1994,1996,1998)$ hace una caracterización semejante de los estilos discursivos de las mujeres y de los hombres, en la que subraya los rituales de competición característicos de la interacción entre hombres de diversas culturas, basados en 'teasing, playfully insulting each other, or playing 'devil's advocate' to develop and strengthen ideas (through, for example, challenges, counter-challenges, and debate" (Tannen 1998: 196); en contraste, esta autora relaciona la interacción femenina con los rituales de apoyo y solidaridad, basados en compartir problemas personales para acentuar las similitudes, la intimidad y la "conexión" entre las participantes (Tannen 1990, 1991; véase también Kendall y Tannen 2001: 553).

\section{LA REALIZACIÓN DE IDENTIDADES DE GÉNERO EN LA INTERACCIÓN}

En la segunda mitad de la década de los noventa, la búsqueda de "diferencias de género" en la interacción comenzó a ser muy criticada, alegando que conlleva una generalización excesiva y que perpetúa los estereotipos socioculturales sobre las

Las tag questions o preguntas coletilla son formaciones sintácticas a medio camino entre las afirmaciones y las preguntas generales de sí o no que pueden utilizarse para pedir confirmación, como en "Miguel está, ¿no?", o para entablar conversación, como en "qué calor hace aquí, ¿verdad?". Lakoff $(1975,2004)$ argumentó que las mujeres suelen utilizarlas para expresar opiniones, como por ejemplo en "la guerra de Irak es terrible, ¿no?”, y que este tipo de usos transmiten inseguridad, porque atenúan la fuerza aseverativa del enunciado. Según esta autora, el empleo de partículas como supongo o creo causa el mismo efecto y es también especialmente característico de las hablantes femeninas. 
formas de hablar de las mujeres y los hombres (Bing y Bergvall 1996; West, Lazar y Kramarae 1997, 2000; Mills 2003; Sunderland 2006). Resaltando la importancia de estos problemas, a partir de entonces comienza a defenderse la necesidad de estudiar el género desde una perspectiva mucho más dinámica, basada en las teorías sobre la construcción social de la realidad (Berger y Luckmann 1966, 1976), en las concepciones del discurso y de la performatividad de Foucault (1972) y Butler (1990, 2001), así como en la noción de "hacer género", procedente del enfoque etnometodológico de West y Zimmermann (1987). A lo largo de los últimos años, las publicaciones en las que se defienden y/o se aplican estos cambios teóricos no han dejado de incrementarse (Crawford 1995; Hall y Bucholtz 1995; Bergvall, Bing y Freed 1996; Johnson y Meinhof 1997; Livia y Hall 1997; Bucholtz, Liang y Sutton 1999; Baron y Kotthoff 2002; Mills 2003; Coates 2003, 2013; Holmes 2006; Pichler 2009; Motschenbacher 2010; Nakamura 2014).

En este marco constructivista/performativo se parte de la idea de que en todas las sociedades y culturas se establecen unos modelos de "feminidad" y de "masculinidad" que las mujeres y los hombres pueden reproducir en su forma de actuar y de hablar, pero también transformar y/o desafiar en mayor o menor medida. La idea clave es que la masculinidad y la feminidad no son algo que se posee, sino algo que se hace, se construye, se realiza o se despliega en la interacción, y además de muy diversas formas, dependiendo de la situación comunicativa y de otros aspectos de la identidad social como la edad, la clase social, el origen cultural o la orientación sexual. Se habla entonces de masculinidades y feminidades, es decir, de múltiples formas de identidades femeninas y masculinas, frente a la visión binaria de la identidad de género que se había asumido hasta entonces en las aproximaciones previas de la dominación y la diferencia. Las investigaciones que proyectan esta nueva perspectiva destacan así, por ejemplo, la construcción de masculinidades juveniles (Coates 2003), de masculinidad heterosexual (Cameron 1997; Coates 2003), de masculinidad simplificada (Pujolar 2002) o de diferentes versiones de feminidad en grupos de chicas de distinta clase social y origen cultural (Pichler 2009).

Por otra parte, se presta especial atención a los casos en los que los hablantes emplean estilos de habla e interacción que están asociados con el "sexo opuesto", interpretándolos como estrategias de adaptación al contexto específico (Holmes 2006) o como prácticas subversivas o transgresoras (Bucholtz 1999; Acuña Ferreira 2012; Nakamura 2014). En la consideración de estos estilos de habla e interacción que están impregnados de género, los estudios previos de la dominación y la diferencia siguen resultando muy útiles, en la medida en que describen numerosos aspectos discursivos que están ligados estereotípicamente con las mujeres y la feminidad o con los hombres y la masculinidad (Holmes 2006: 5-9; véase también Motschenbacher 2010: Cap. 4). Además, se resaltan situaciones en las que los hablantes cuestionan los modelos predominantes de género de manera explícita, en el nivel de contenido del discurso, como una forma de explorar identidades alternativas. Por ejemplo, el libro de Jennifer Coates (2003), Men talk, en el que se analiza un corpus de charlas amistosas entre hombres británicos de distintas edades y clases sociales, incluye el análisis de secuencias en las que varios participantes debaten sobre la necesidad de compartir información personal e íntima con los demás, en contra de las expectativas socioculturales que hacen incompatible la expresión de sentimientos con el concepto de "ser un hombre" (2003: 73-8). En sus conclusiones, no obstante, Coates enfatiza 
que estos desafíos no son lo habitual y que, en general, las interacciones masculinas de su corpus contribuyen a reforzar los modelos convencionales de masculinidad: "in most of the conversations most of the time, it is evident that male speakers are acting in a way that aligns them with these dominant norms, norms which prescribe 'acceptable' maleness" (2003: 196).

\subsection{Las comunidades de práctica}

Eckert y McConnell-Ginet (1992) presentan una extensa revisión crítica de las investigaciones sobre Lengua y Género realizadas hasta la fecha, en la que defienden las ventajas que presenta la noción de Comunidad de Práctica (Lave y Wenger 1991), para la aplicación del enfoque constructivista/performativo. La Comunidad de Práctica se define como "an aggregate of people who come together around mutual engagement in an endeavour. Ways of doing things, ways of talking, beliefs, values, power relations -in short, practices- emerge in the course of this mutual endeavour" (Eckert y McConnell-Ginet 1992: 464; véase también Eckert y McConnell-Ginet 1999). Este concepto permite abordar el estudio de las identidades de género de forma localizada, explicando las diferentes versiones de feminidad y de masculinidad que pueden desplegar las personas en sus interacciones cotidianas en relación con las normas, los valores y las actitudes específicas que se fomentan en su pandilla de amigos, en el lugar donde trabajan o en su círculo familiar (véase también Pujolar 2002, 2003; Holmes 2006). Uno de los primeros estudios que aplicaron este enfoque es el de Bucholtz (1999), que analiza las prácticas de identidad de un grupo de estudiantes de un instituto de California que se identifican como nerd girls ("chicas intelectuales"). Bucholtz demuestra que esta identidad intelectual se construye en gran parte como una forma de desafiar los ideales de feminidad o la feminidad hegemónica, frente a otros grupos de chicas, cuyas prácticas identitarias, en cambio, reproducen esos modelos sociales predominantes: "For girls, nerd identity also offers an alternative to the pressures of hegemonic feminity -an ideological construct that is at best incompatible with, and at worst hostile to, female intellectual ability" (Bucholtz 1999: 213).

Más recientemente, Holmes (2006) analiza las identidades de género en la comunicación en el lugar de trabajo desde una perspectiva constructivista y performativa, basándose en un corpus de interacciones reales obtenidas en diversos centros de trabajo de Nueva Zelanda y aplicando también el concepto de Comunidad de Práctica, que considera muy útil para comprender por qué se dice a menudo que algunos lugares de trabajo tienen una cultura laboral "masculina" o "femenina" (Holmes 2006: 13). Las formas de hablar y de interactuar desempeñan un papel muy importante a este respecto, pues en algunos centros predominan los estilos estereotípicamente masculinos (directos, competitivos y centrados en la función referencial del lenguaje), mientras que en otros es el discurso estereotípicamente femenino el que funciona como la variedad no marcada (indirecto, colaborativo y centrado en la dimensión afectiva del lenguaje). Su análisis pone de relieve cómo estos estilos son empleados tanto por hombres como por mujeres, dependiendo de la comunidad de práctica de la que forman parte y de otros factores contextuales como el tipo de interacción, las personas que participan en la misma o el contexto discursivo específico (véase también Acuña Ferreira 2009b). 


\subsection{Críticas a los estudios constructivistas/performativos}

La aplicación empírica del enfoque constructivista/performativo de la identidad de género ha recibido críticas desde el Análisis Conversacional (AC). Basándose en las observaciones de Sacks (1992) sobre el análisis de las identidades en la conversación, Schegloff (1997) ha argumentado que, para poder explicar lo que sucede en cualquier interacción en relación con el poder y/o el género, es necesario que puedan observarse señales explícitas de que los participantes están hablando "como hombre" o "como mujer" y de que esta identidad específica tiene consecuencias para el desarrollo de la actividad comunicativa. En los últimos años se han publicado numerosos trabajos en los que se defienden estas bases del AC para el estudio de las identidades en general y de las identidades de género en particular, criticando no solo las aproximaciones iniciales de la dominación y la diferencia, sino también la metodología analítica de los estudios constructivistas/performativos. Sobre estos últimos, se considera que "rely heavily on analysts assumptions about what the speakers are doing rather than on what the speakers display to each other as relevant to their conversational business" (Benwell y Stokoe 2005: 57).

Algunas autoras han respondido a estas críticas afirmando que el marco del AC resulta demasiado restrictivo, porque "limits admissible context so severely that only the most blatant aspects of gendered discursive practice, such as the overt topicalizing of gender in conversation, are likely candidates for Schegloffian analysis" (Bucholtz 2003: 52). Los posicionamientos de ambas aproximaciones han sido objeto de un intenso debate dentro del campo y actualmente se desarrollan de forma paralela, configurando dos áreas sólidas y bien diferenciadas. Desde finales de los años noventa, cada vez son más las investigaciones que defienden y aplican la metodología del AC (véase Speer y Stokoe 2011a), frente a la perspectiva de aquellas investigadoras que, desde la Sociolingüística y otras corrientes del Análisis del Discurso, "simply do not accept that social categories need to be observably and explicitly salient for participants in order to be considered relevant in their analyses" (Holmes 2007: 54).

\section{LA REALIZACIÓN DE FEMINIDAD ENTRE JÓVENES VEINTEAÑERAS}

\subsection{Objetivos generales y especificos}

En los apartados anteriores hemos presentado el estado de la cuestión en los estudios sobre el género en la interacción, destacando las diferentes corrientes teóricometodológicas que han marcado su trayectoria hasta el momento. En este apartado vamos a centrarnos en el análisis de datos conversacionales desde una perspectiva de género, apoyándonos en los fundamentos teóricos de las aproximaciones constructivistas/performativas y en la noción de Comunidad de Práctica (Eckert y McConnell-Ginet 1992, 1999; véase el apartado 2.1), de acuerdo con lo explicado en esa revisión previa. Situándonos dentro de este marco teórico-metodológico, nuestro objetivo es presentar un estudio de caso sobre la realización de identidades de género en la conversación, analizando extractos de una de las interacciones de nuestro corpus conversacional (Acuña Ferreira 2009a, 2011; véase la Sección 3.2.). Se trata de una conversación entre tres jóvenes veinteañeras, en la que la actividad comunicativa se 
basa en compartir confidencias sobre las relaciones sexuales/afectivas con los hombres y en la que el género adquiere, por tanto, un papel central. Conviene subrayar que, en el enfoque constructivista/performativo que adoptamos, el género no se asume como una categoría social omnipresente, que tiene relevancia en cualquier situación y que siempre se manifiesta de la misma forma, sino que se entiende como una realización estrechamente ligada al contexto y enormemente variable en función del mismo. Esta perspectiva invita a las investigaciones a identificar los contextos comunicativos en los que se despliega o se realiza el género y a analizar cómo se desarrolla este proceso, teniendo en cuenta la comunidad de práctica específica (véase Acuña Ferreira 2009a).

Así pues, la razón principal por la que centramos nuestro estudio en esta conversación se debe a que se basa en una actividad comunicativa relacionada con los hombres y con las relaciones heterosexuales, por lo que representa un acontecimiento conversacional en el que el género adquiere gran importancia. Además, se trata de una conversación juvenil, que nos permite abordar la interacción de esta categoría social con la edad. Desde la perspectiva del AC (véase la Sección 2.2.), estos datos conversacionales también podrían analizarse como ejemplo de la relevancia del género en la interacción, pues las jóvenes participantes se identifican abiertamente como mujeres heterosexuales al compartir este tipo de confidencias. Sin embargo, como hemos señalado, nuestra aproximación a esta interacción se apoyará en el enfoque constructivista/performativo. Al adoptar esta perspectiva, es importante resaltar que nuestra valoración de los datos no se limita a un análisis "técnico" de los mismos, como se reivindica desde el $\mathrm{AC}$, sino que también incluye la observación etnográfica y nuestro trabajo interpretativo como analista, basado en nuestro conocimiento académico, social y cultural del género (véase también Pichler 2009: 12-13). Por ello, nuestro estudio va más allá de ese proceso de identificación como mujer heterosexual y se propone, de manera más específica, analizar extractos de esta conversación natural como muestra de la realización de feminidades juveniles en un grupo de amigas y del papel destacado que cumple a este respecto el intercambio de confidencias sobre las relaciones sexuales/afectivas con los hombres, como acontecimiento conversacional impregnado de género ${ }^{2}$.

\subsection{Datos y metodología}

En la conversación que vamos a analizar participan tres mujeres de edades comprendidas en torno a los veinticinco años, a las que llamaremos Eva, María y Cris $^{3}$. Las tres se conocen desde hace años y durante este tiempo han formado un grupo sólido de amigas, junto con otras chicas, que se reúnen cada verano en una pequeña ciudad de Galicia, España, donde viven sus padres. Algunas de estas chicas son naturales de esta localidad y viven en ella todo el año, mientras que otras estudian o trabajan en otras ciudades gallegas o españolas y solo se trasladan aquí en época de

La realización de feminidad y/o de masculinidad se define como objetivo principal en las aproximaciones constructivistas/performativas. Los estudios sobre género que se sitúan dentro del marco del AC no suelen trabajar con los conceptos de "feminidad" o "masculinidad" y formulan sus preguntas de investigación en otros términos (véase Speer y Stokoe 2011b).

Empleamos siempre seudónimos, tanto en el caso de los participantes como en el de las terceras personas de las que se habla en las interacciones. 
vacaciones. Este grupo de amigas constituye una de las comunidades de práctica en las que se centró el trabajo de campo etnográfico que realizamos para la formación de un corpus de conversaciones cotidianas, como base para nuestras investigaciones sobre género y discurso (Acuña Ferreira 2009a, 2011). Nuestro corpus está constituido por interacciones naturales que fueron grabadas en audio a través de la observación participante en la comunicación de grupos de amigos, familiares y compañeros de clase o de trabajo, es decir, en diferentes tipos de comunidades de práctica. Algunas de estas interacciones fueron extraídas de un corpus mucho más amplio, el Corpus de Fala Bilingüe Galego/Castelán (Co.Fa.Bil) de la Universidad de Vigo (véase Rodríguez Yáñez y Casares Berg 2002-2003).

Todas las integrantes de este grupo de amigas aceptaron colaborar en nuestro proyecto, dando permiso para la grabación de algunas de sus interacciones, e incluso grabándose ellas mismas. Para lograr la máxima espontaneidad posible, se pidió a las colaboradoras que no avisasen previamente a las demás de la grabación que se iba a realizar y que informasen de la misma después de haberla efectuado, pidiendo permiso para entregarla al proyecto en curso.

La conversación de Eva, María y Cris en la que vamos a centrarnos fue grabada en una bocatería, en la que estas tres amigas decidieron reencontrarse, después de varios meses sin haberse visto, poco después del comienzo de las vacaciones de verano. Durante los casi 30 minutos que dura la grabación, la actividad comunicativa se centra en las experiencias que Eva ha compartido recientemente con un chico, a quien conoció en la ciudad donde está realizando estudios universitarios y donde vive la mayor parte del año. Esta participante cuenta numerosas historias en las que ofrece detalles sobre la forma en que comenzó esta relación y cómo ha ido desarrollándose con el tiempo, posicionándose de este modo como hablante principal, mientras que las demás tienden a actuar como hablantes secundarias (Bublitz 1988), indicando atención, aportando su opinión o compartiendo una historia acerca de su propia relación de pareja, que guarda relación con alguno de los subtemas que van surgiendo ${ }^{4}$. Así, la interacción puede describirse en general como una sesión de confidencias sobre las relaciones heterosexuales, las cuales son analizadas especialmente a través de las experiencias contadas por Eva. Este tipo de acontecimiento comunicativo era bastante común entre las integrantes de este grupo de amigas y puede considerarse representativo del tipo de interacciones que lo caracterizan como una Comunidad de Práctica.

La transcripción de los datos se hizo de acuerdo con una serie de convenciones que, en conjunto, tratan de plasmar la mayor cantidad posible de información sobre el desarrollo del habla, incluyendo fenómenos prosódicos, de tal forma que su lectura no resulte demasiado complicada. Para ello nos hemos apoyado en el sistema de Álvarez Cáccamo (Véase el Anexo que se incluye al final del artículo, para conocer cada una de estas convenciones; véase también Acuña Ferreira 2009a: 9-23).

\subsection{Análisis de extractos conversacionales}

Desde los primeros momentos de la conversación, Eva comenta que ha estado saliendo y enrollándose con un chico, pero deja claro que no se trata de su novio

\footnotetext{
En esta conversación intervienen especialmente Eva y Cris. La participación de María es mucho menos notable en una grabación de audio, quizás debido en parte a que es la autora de la misma.
} 
o su chorbo. De este modo se alude implícitamente a dos modelos de relaciones heterosexuales que están ligados al estilo de vida de las generaciones más recientes. Algunos estudios sociológicos sobre la juventud española han destacado importantes cambios en el estilo de vida sexual con respecto a generaciones anteriores, como la existencia de una clara distinción entre "relaciones de noviazgo", en las que existe un compromiso de fidelidad mutua y se valora el compañerismo, frente a las "relaciones informales", en las que no se establece ese compromiso y "se entiende que la mujer también dispone libremente de su cuerpo y de su sexualidad, y puede dar salida a sus deseos y pulsiones sexuales tan libremente como los hombres" (Megías y Ballesteros 2014: 188).

Eva sitúa sus experiencias con el chico dentro de este último modelo de relación, pero a lo largo de la charla va contando numerosas historias acerca de la misma en las que destaca los vínculos de afecto, de complicidad y de amistad que han surgido entre ambos con alegría y entusiasmo, como señales de que la situación podría evolucionar hacia un noviazgo, mientras que las demás se afilian con ella, indicando su deseo de que esto sea así, aportando su opinión, dando consejo o compartiendo una historia relacionada con alguna de las cuestiones que se van tratando.

Uno de los momentos que resulta más significativo a este respecto se produce cuando Eva cuenta una historia acerca de algo que sucedió cuando hablaba con el chico durante el último encuentro que tuvo con él, poco antes de las vacaciones. El Extracto 1 recoge la parte central de esta historia:

\section{Extracto 1}

\begin{tabular}{|l|l|l|}
\hline 222 & Eva & pero yo muchas cosas $\uparrow$ \\
\hline 223 & & me corto $\rightarrow$ \\
\hline 224 & & al decirle lo que siento $\rightarrow$ \\
\hline 225 & & {$[(\mathrm{xx})]$} \\
\hline 226 & Cris & {$[(\mathrm{xx})]$} \\
\hline 227 & & yo eso no lo entiendo tía $\uparrow$ \\
\hline 228 & & $<0.5>$ \\
\hline 229 & Eva & pues me co:rto tía $\uparrow$ \\
\hline 230 & & me corto $\uparrow$ \\
\hline 231 & & porque me corto $\rightarrow$ \\
\hline 232 & & no? \\
\hline 233 & & y yo $\rightarrow$ \\
\hline 234 & & y le dije $\rightarrow$ \\
\hline 235 & & jo:: $\uparrow$ \\
\hline 236 & & te voy a echar más de me:nos $\uparrow<$ con voz melosa $>$ \\
\hline 237 & & y tal $\uparrow<$ entre risas $>$ \\
\hline 238 & $<1>$ \\
\hline 239 & & es que no me dijo $\uparrow$ \\
\hline 240 & & ni pala:bra tía $\rightarrow$ \\
\hline 241 & & \\
\hline
\end{tabular}




\begin{tabular}{|l|l|l|}
\hline 242 & & pero \\
\hline 243 & & se me quedó viendo \\
\hline 244 & & $($ xx $)$ \\
\hline 245 & & sabes? $=$ \\
\hline 246 & Cris & $=$ ahá \\
\hline 247 & Eva & y \\
\hline 248 & & y se $=$ \\
\hline 249 & Cris & $=$ todo tuyo Eva $\uparrow$ \\
\hline 250 & & {$[$ he he he $]$} \\
\hline 251 & Eva & {$[$ Y BAJÓ LA] CABEZA $\uparrow<$ entusiasmada $>$} \\
\hline 252 & & ASÍ EN PLAN $\rightarrow<$ entusiasmada $>$ \\
\hline 253 & & $<1.5>$ \\
\hline 254 & & sabes? $=$ \\
\hline 255 & Cris & $=$ sí $=$ \\
\hline 256 & Eva & $=$ ES QUE A MÍ NO ME $<$ entusiasmada $>$ \\
\hline 257 & & NO ME DICE NADA $\uparrow<$ entusiasmada $>$ \\
\hline 258 & & PERO ES QUE $\rightarrow<$ entusiasmada $>$ \\
\hline 259 & & {$[$ LUEGO PONE CARAS Y CON GESTOS] $\uparrow<$ entusiasmada $>$} \\
\hline 260 & Cris & {$[$ y se nota que:: ya] } \\
\hline 261 & Eva & {$[$ que me lo dicen todo] $\downarrow=$} \\
\hline 262 & Cris & $=\{[$ p] que lo dicen todo $\} \downarrow$ \\
\hline 263 & & .. \\
\hline 264 & & sí \\
\hline 265 & & (pues a ver si:: $)=$ \\
\hline 266 & Eva & $=$ sabes? \\
\hline & & \\
\hline
\end{tabular}

La historia se basa en la reconstrucción de una escena de despedida en la que se produce una tímida demostración de sentimientos por parte de Eva y el chico. Antes de reconstruir esta escena, a modo de orientación de la historia (Labov y Waletzky 1967; Labov 1972), Eva comenta que suele contenerse a la hora de expresar sentimientos cuando está con él y esto da lugar a una breve discusión, ya que Cris expresa asombro por esta actitud, mientras que Eva insiste en que esto es algo que le produce vergüenza (líneas 222-232). Zanjando la discusión, la narradora-protagonista retoma la historia y reconstruye en estilo directo sus propias palabras diciéndole al chico que le echaría de menos, recreando una voz "melosa" (líneas 234-237). Seguidamente describe la reacción de él, señalando que no dijo nada, pero destacando cómo la miró, y apelando a sus interlocutoras para comprobar que entienden lo que quiere decir (líneas 240-245). Cris asiente inmediatamente (línea 246) y además "celebra" esa mirada del chico en clave de humor como señal de que su amiga ha logrado conquistarlo (líneas 249-250).

En los siguientes turnos, Eva elabora su descripción utilizando un volumen de voz más alto que transmite su entusiasmo ante los hechos: resalta el movimiento que hizo el chico con la cabeza (líneas 251-252) y comenta este tipo de respuestas gestuales como algo habitual en él, utilizando verbos en presente como dice y 
pone (líneas 256-259). Cambiando a un volumen de voz "normal", Eva concluye destacando la importancia de estos gestos como señales muy reveladoras, que me lo dicen todo (línea 261), acerca de la reciprocidad de sentimientos que existe dentro de la relación. Cris refuerza la "conexión" con su amiga, confirmando que la comprende o que comparte su interpretación de lo sucedido (líneas 254-255), completando o repitiendo sus enunciados (líneas 260,262) y expresando su deseo de que la relación se consolide o siga por este camino (línea 265).

A través de la narración de esta historia, Eva aborda la expresión de sentimientos como algo delicado en la relación que está manteniendo (al confesar que esto le produce vergüenza), pero también demuestra interés en traspasar esta línea y entusiasmo ante las señales de reciprocidad emocional que recibe. No expresa desacuerdo cuando su amiga Cris apoya su interpretación de los hechos que reconstruyen la historia como una demostración de afecto por parte del chico, y además manifiesta de forma bastante explícita su deseo de que la relación vaya consolidándose.

Todo ello indica que las participantes hablan de esta "relación informal" como una oportunidad de formar una relación de pareja o como una etapa de transición hacia ella. Nótese, por otra parte, que el chico queda representado en el mundo de la historia de acuerdo con la imagen del hombre silencioso que no verbaliza sus sentimientos, un estereotipo de género que puede explicarse como resultado de las normas de restricción emocional que establecen los ideales de masculinidad (Coates 2003). En cambio, Eva despliega feminidad al ser quien toma la iniciativa en este sentido, dentro del mundo de la historia.

En una fase más avanzada de la charla, Eva comenta cómo es la personalidad del chico, explicando que este le dijo que podía interesarse o preguntarle por su vida, pero sin "meterse" en ella. Su amiga Cris interpreta que esta es una forma de salvaguardar su independencia personal dentro de la relación y cuenta una historia sobre algo que le sucedió a ella con su propia pareja. El Extracto 2 recoge una primera parte de esta historia de Cris:

\section{Extracto 2}

\begin{tabular}{|l|l|l|}
\hline 686 & Cris & {$[($ yo es una $)]$ etapa $\uparrow$} \\
\hline 7 & & que viví con Javi $\rightarrow$ \\
\hline 8 & & $($ yo sé que fue) un día \\
\hline 9 & & $($ xx $)$ \\
\hline 0 & & ah $\uparrow<$ voz simulada $>$ \\
\hline 1 & & porque yo cuando acabe:: $<$ voz simulada $>$ \\
\hline 2 & & cuando:: $<$ voz simulada $>$ \\
\hline 3 & & acabe la carrera $\uparrow<$ voz simulada $>$ \\
\hline 4 & & pues quiero hacerme $(\mathrm{xx})<$ voz simulada $>$ \\
\hline 5 & & $(\mathrm{xx})$ me voy a ir de caravana:: $<$ voz simulada $>$ \\
\hline 6 & & $(\mathrm{xx})$ \\
\hline 7 & & la típica callada $=$ \\
\hline 8 & Eva & $=$ sí $=$ \\
\hline 9 & Cris & $=$ que dices $\uparrow$ \\
\hline 0 & & .. \\
\hline
\end{tabular}




\begin{tabular}{|c|c|}
\hline 1 & tía $\rightarrow$ \\
\hline 2 & .. \\
\hline 3 & es que estás comentando \\
\hline 4 & pues? \\
\hline 5 & normalísimo $\rightarrow$ \\
\hline 6 &.. \\
\hline 7 & y a mí me sentó fatal $\uparrow$ \\
\hline 8 & el sentirme $\rightarrow$ \\
\hline 9 & joder tío $\rightarrow$ \\
\hline 0 & cuando acabes $\uparrow$ \\
\hline 1 & qué coño $\rightarrow$ \\
\hline 2 & yo me voy a vivir contigo $\uparrow$ \\
\hline 3 & qué caravana $\uparrow$ \\
\hline 4 & ni qué hostias $\rightarrow$ \\
\hline 5 & eso será en verano $\uparrow$ \\
\hline 6 &.. \\
\hline 7 & $(\mathrm{xx})$ \\
\hline
\end{tabular}

Esta historia de Cris se basa en la reconstrucción de un diálogo entre ella y su novio (identificado como Javi en la transcripción), que la narradora-protagonista introduce como un episodio representativo de determinada etapa dentro de esta relación y ofreciendo pocos datos de orientación (líneas 686-689). La producción de la historia avanza rápidamente hacia la fase de complicación (Labov y Waletzky 1967; Labov 1972), que se inicia con la recreación de la voz del novio anunciando su intención de hacer un viaje en caravana tras finalizar sus estudios universitarios (líneas 690-695). La narradora-protagonista sugiere que estos comentarios dieron lugar a un silencio incómodo porque la dejaron boquiabierta, apelando a sus interlocutoras por medio de una pregunta para suscitar su complicidad o invitarlas a afiliarse con su propia reacción (línea 697). Eva asiente para indicar su atención (línea 698) y entonces Cris trata de explicar cómo le hicieron sentir esas palabras de su pareja, haciendo breves pausas que contribuyen a representar sus dificultades para reaccionar ante las mismas y apelando nuevamente a su interlocutora, a través del uso del vocativo tía (líneas 699-702). Seguidamente retoma la reconstrucción del diálogo, introduciéndolo directamente, sin emplear verbos de decir ni realizar cambios en la calidad de la voz (líneas 703-704). En esta breve recreación del discurso en el mundo de la historia, la narradora-protagonista reproduce sus propias palabras indicando desconcierto y la réplica de su novio con una pregunta que indica su falta de entendimiento ante esa reacción de ella (líneas 703-704). El enunciado de la línea 705 parece un comentario que la narradora hace dentro del contexto narrativo para remarcar la actitud de normalidad que mostraba el chico en ese diálogo de manera explícita, en contraste con su estado de confusión o sorpresa como protagonista femenina ${ }^{5}$.

\footnotetext{
El enunciado normalísimo podría formar parte del discurso del personaje masculino en el diálogo reconstruido, pero es imposible asegurarlo debido a la ausencia de marcas que indiquen claramente los límites de esa reconstrucción.
} 
Tras una pausa (línea 706), Cris declara explícitamente lo mal que le sentaron esas palabras de su novio (línea 707) y reivindica su intención de irse a vivir con él en un futuro, rechazando enérgicamente sus planes de viajar en caravana, a través del empleo de lenguaje ordinario o de expresiones tabú como joder, qué coño, ni qué hostias, que despliegan su indignación de forma muy poderosa (líneas 708-715). Resulta difícil asegurar si esta reacción afectiva es algo que la narradora-protagonista introduce como las palabras que pronunció en respuesta a su pareja, o como algo que pensó en ese momento, ya que este segmento está enmarcado por el verbo sentir (línea 708), y no por verbos de decir o por otros que indiquen pensamiento de forma clara.

Nos inclinamos por considerar que se trata de un caso de pensamiento referido que ha sido introducido de manera ambigua, de acuerdo con Haakana (2007), que destaca esta falta de claridad como algo frecuente en su análisis de los usos de este tipo de discurso en las historias de queja (Günthner 1997; Acuña Ferreira 2011). Además, el análisis de esta autora demuestra que el pensamiento referido en la producción de este tipo de historias se articula a menudo como una respuesta mental del protagonista a la tercera persona de la que se queja y que esta forma de crítica silenciosa suele tener una carga afectiva muy intensa, mayor que la de las respuestas verbales que son representadas como discurso referido ${ }^{6}$. Como en los casos analizados por esta investigadora, en este extracto Cris emplea esta respuesta mental cargada de indignación como un recurso de evaluación implícita de los hechos, que invita a sus interlocutoras a expresar afiliación con su perspectiva crítica ante los mismos y que contribuye de forma esencial a construir la historia como una historia de queja (Haakana 2007; véase también Günthner 1997; Acuña Ferreira 2011).

En el Extracto 3, que recoge la parte final de la historia, Cris representa la respuesta verbal que dio a su novio, enmarcándola claramente como discurso referido directo:

\section{Extracto 3}

\begin{tabular}{|l|l|l|}
\hline 6 & Cris & tía $\uparrow$ \\
\hline 7 & & lo típico $\uparrow$ \\
\hline 8 & & que ya te haces tu:: \\
\hline 9 & & joder es verdá? \\
\hline 0 & .. \\
\hline 1 & $\{[$ p] y el tío con cara de $(\mathrm{xx})\} \downarrow$ \\
\hline 2 & $(\mathrm{xx})$ \\
\hline 3 & tía me dolió $\rightarrow$ \\
\hline 4 & sabes? \\
\hline 5 & porque (le dije) \\
\hline 6 & & ah muy bien $\rightarrow$ \\
\hline 7 & tío $\rightarrow$ \\
\hline 8 & o sea \\
\hline
\end{tabular}

\footnotetext{
Haakana (2007: 176) considera que la ambigüedad con la que se introduce en muchos casos un pensamiento referido cumple a menudo una función: "the narrator can imply that he or she actually criticised the antagonist or fought back in the situation but does not have to claim this in more explicit terms (such as with a reporting utterance, 'I said')".
} 


\begin{tabular}{|l|l|l|}
\hline 9 & & tú acabas $\uparrow$ \\
\hline 0 & & y a tomar por culo sabes? \\
\hline 1 & & (o sea la típica) \\
\hline 2 & & LA TÍPICA:: \\
\hline 3 & & $(\mathrm{xx})$ \\
\hline 4 & & PERO QUE A TI TE JODE $\uparrow$ \\
\hline 5 & & porque te jode $\downarrow$ \\
\hline 6 & & tía $\rightarrow=$ \\
\hline 7 & Eva & $=$ te mueres $=$ \\
\hline
\end{tabular}

Aquí Cris explica su indignación indicando que ella había pensado en un futuro junto a su novio, y no en solitario o con amistades, como presumiblemente había planteado él (líneas 718-721). Al ofrecer estas explicaciones, la hablante apela a sus interlocutoras para solicitar su afiliación o empatía a través de diversas estrategias: emplea de nuevo el vocativo tía (línea 718), habla en segunda persona de singular para generalizar sus pensamientos o su forma de sentir y de experimentar la relación (línea 720; véase Vila 1987) y emplea una entonación ascendente, típicamente interrogativa (línea 721). Hasta el momento, ninguna de las interlocutoras ha expresado esa afiliación de forma verbal, lo que puede explicar en parte que el discurso esté tan marcado por este tipo de estrategias $^{7}$. Tras una breve pausa (línea 722), la narradora-protagonista vuelve a resaltar la actitud de normalidad o indiferencia de su novio, esta vez de forma muy negativa, refiriéndose a él despectivamente como el tío (línea 723). A continuación, expresa una vez más su malestar de forma explícita, utilizando el verbo doler (líneas 725-726), y reconstruye cómo le reprochó sarcásticamente su intención de dejar supuestamente la relación al finalizar sus estudios (líneas 727-732), empleando el verbo decir para enmarcar estas palabras como discurso referido directo, es decir, como una respuesta verbal y no como algo que pensó. De acuerdo con lo observado por Haakana (2007), esta respuesta verbal no se presenta tan cargada afectivamente como sucedía en el caso de la respuesta mental que se articuló previamente, como vimos en el Extracto 3.

Finalmente, Cris hace una evaluación explícita de las palabras de su novio, que resulta parcialmente ininteligible (líneas 733-735), y enfatiza retóricamente el daño que estas le produjeron, empleando el verbo joder y fórmulas de apelación a sus interlocutoras, como los pronombres de segunda persona de singular ( $a$ ti, te) para generalizar ese sentimiento y suscitar complicidad (Vila 1987), así como el vocativo tía en la finalización del turno (líneas 736-738). Eva responde de forma afiliativa, utilizando el verbo morirse en segunda persona de singular para hacerse eco del enunciado de su amiga y reforzar su solidaridad con ella (línea 739). En general, todo el discurso de Cris está muy marcado por el uso de técnicas de apelación al interlocutor; en este sentido destacan especialmente los usos reiterados de tía y de ¿sabes? (líneas 718, 725-726, 732, 738).

Es posible que las interlocutoras hayan desplegado afiliación a través de expresiones faciales que solo se podrían observar en una grabación de video. La insistencia de la hablante en remarcar su malestar, en ofrecer explicaciones y en utilizar fórmulas de apelación sugiere que estas señales no se consideran suficientes. 
En los turnos que siguen a los recogidos en este extracto, las jóvenes debaten un poco sobre esta historia de Cris e interpretan las palabras de su novio como una forma de marcar su espacio personal dentro de la relación, pero no vamos a analizar esa secuencia posterior de comentarios sobre los hechos. Por razones de espacio, ponemos el foco en la narración de esta experiencia por parte de Cris como muestra del alto nivel de intimidad que se crea cooperativamente en esta interacción, a través del intercambio de confidencias personales, que desempeñan un papel central en la construcción de un estilo de comunicación femenino (Jones 1980; Johnson y Aries 1983; Tannen 1990, 1991; Coates 1996). Como en la historia de Eva que analizamos antes (en el Extracto 1), esta historia de Cris hace hincapié en los sentimientos, aunque en este caso se trata de quejas por una supuesta indiferencia o falta de implicación del chico y no de una "celebración" por sus demostraciones de afecto. Así, la narradoraprotagonista describe explícitamente el daño sentimental que le produjo la actitud de su novio, a través de expresiones como me dolió, me sentó fatal, el sentirme o te jode, pero también hace un despliegue de indignación muy intenso, en el que destaca el uso de lenguaje ordinario o expresiones malsonantes.

El uso de este tipo de expresiones no es algo exclusivo de Cris, sino que es común a las tres participantes. El lenguaje ordinario ha estado ligado tradicionalmente al habla de los hombres y a la masculinidad ${ }^{8}$, pero De Klerk (1997) sugiere que esto está cambiando entre las nuevas generaciones, ya que en su investigación no encontró grandes diferencias en el uso de tacos por parte de los chicos y las chicas de una escuela de habla inglesa de Sudáfrica ${ }^{9}$. Así pues, consideramos que el empleo de este tipo de lenguaje en este grupo de chicas puede interpretarse como una forma de marcar su identidad femenina específica como mujeres jóvenes, es decir, como una forma de construir una feminidad juvenil, en la que el uso de tacos se acepta y se utiliza como símbolo de igualdad con respecto a los hombres.

Por último, es importante resaltar cómo se "hace género" (West y Zimmermann 1987) dentro del mundo que aparece representado en esta historia contada por Cris. La narradora-protagonista hace un despliegue de indignación intenso, cargado de expresiones "fuertes", que construyen un discurso masculinizado y estereotípicamente "poderoso" (Lakoff 1975, 2004). Sin embargo, este despliegue de indignación se sitúa dentro del mundo de la historia como una respuesta mental de la protagonista, como algo que esta pensó en ese momento, pero que no dijo, y que probablemente reprimió en el contexto original. En general, tanto el personaje femenino como el masculino aparecen representados de forma muy convencional, de acuerdo con los estereotipos sobre las relaciones heterosexuales: ella se muestra muy implicada en esa relación de pareja como un proyecto de vida en común y se siente herida por los planes de futuro que comenta él, aparentemente sin contar con ella. Mientras que en la historia previa de Eva, el personaje masculino reproducía el estereotipo del hombre silencioso, aquí el chico queda representado de acuerdo con el estereotipo del hombre

Véase Coates (2003) sobre el uso de "lenguaje tabú" como realización de masculinidad en las historias contadas por hombres. Acuña Ferreira (2002-2003, 2004, 2008, 2009a, 2011) resalta los despliegues de indignación especialmente agresivos que marcan las historias de queja contadas por hombres frente a las de las mujeres, en parte debido al uso de expresiones interjectivas que están muy estigmatizadas.

$9 \quad$ La interacción del género con la clase social también es muy importante a este respecto: Hughes (1992) destaca las expresiones ordinarias empleadas por mujeres británicas de las clases sociales más bajas. 
independiente, que hace planes al margen de su pareja y aparenta cierta indiferencia o falta de implicación emocional con ella y con la relación que ambos mantienen ${ }^{10}$.

Vamos a concluir esta sección de análisis con una última historia contada por Eva, que se cuenta ya en la fase final de la conversación. Se trata de una historia semejante a la que vimos en el Extracto 1 y a otras muchas de las contadas por esta participante, en la medida en que se centra en celebrar otro detalle de afecto por parte del chico con el que mantiene esa relación informal que está en el centro de la actividad comunicativa. En la fase de orientación de esta historia, la narradora-protagonista sitúa los hechos en un momento en el que se encontraba muy sensible porque había llegado la época de exámenes y echaba de menos a sus padres. El Extracto 4 recoge los últimos turnos que comprende esta fase de orientación y toda la reconstrucción de los hechos principales que sigue a continuación:

\section{Extracto 4}

\begin{tabular}{|c|c|c|}
\hline 999 & Eva & o sea que $\rightarrow$ \\
\hline 1000 & & super sensible $\downarrow$ \\
\hline 1001 & & $(\mathrm{xx})$ \\
\hline 1002 & & y yo diciendo $\rightarrow$ \\
\hline 1003 & & jode: $: \mathrm{r} \rightarrow<$ con tono de tristeza $>$ \\
\hline 1004 & & (xx) en todo el dí::a $\uparrow<$ con tono de tristeza $>$ \\
\hline 1005 & & no me llamó:: $\uparrow<$ con tono de tristeza $>$ \\
\hline 1006 & Cris & $=\mathrm{sí}=$ \\
\hline 1007 & Eva & $=\mathrm{y}$ en eso $\uparrow$ \\
\hline 1008 & & pi pi $\uparrow$ \\
\hline 1009 & & mensaje $\rightarrow$ \\
\hline 1010 & &.. \\
\hline 1011 & & mucha suerte $\rightarrow$ \\
\hline 1012 & & un be:so $\downarrow$ \\
\hline 1013 & & he he he $=$ \\
\hline 1014 & Cris & $=$ he he he \\
\hline 1015 & & qué ri:co = \\
\hline 1016 & Eva & $=$ y yo \\
\hline 1017 & & $\mathrm{a}:::: \mathrm{h} \uparrow=$ \\
\hline 1018 & Cris & $=$ he he he $=$ \\
\hline 1019 & Eva & $=0:::::::::::::_{h}$ \\
\hline 1020 & & $<$ simula que está llorando $>$ \\
\hline 1021 & &.. \\
\hline 1022 & & he he he \\
\hline
\end{tabular}

10 La escena que se reconstruye en esta historia se asemeja a muchos de los casos de malentendidos dentro de la pareja heterosexual analizados por Tannen (1990, 1991), que señala el valor de la independencia personal como un elemento clave en el estilo de comunicación de los hombres, frente a la importancia de la expresión afectiva en el caso del estilo comunicativo de las mujeres. 
En los primeros turnos que recoge este extracto, Eva destaca explícitamente lo sensible que estaba el día en que sucedieron los hechos (líneas 999-1000) y representa en estilo directo cómo se quejó de que el chico no la hubiera llamado (líneas 10021005). Esta recreación está introducida por el verbo decir (línea 1002), como si se tratase de discurso referido, pero cabe interpretar que se trata de los pensamientos de la protagonista, y por tanto de un pensamiento referido (Haakana 2007), en la medida en que Cris se refiere al chico en tercera persona y no indica que esté en compañía de nadie. A diferencia de lo que veíamos en el Extracto 3, aquí el pensamiento referido no funciona como un recurso de evaluación, sino como una información contextual, que trata de favorecer una interpretación adecuada del mensaje que se quiere transmitir con la historia y que, por tanto, forma parte de la orientación de la misma.

Tras una respuesta mínima de afirmación de Cris que indica atención al proceso reconstructivo (línea 1006), Eva utiliza la expresión y en eso (línea 1007) para llamar la atención de sus interlocutoras sobre la reconstrucción de los hechos principales. Acto seguido, indica la llegada de un mensaje a su teléfono móvil por medio de onomatopeyas (líneas 1008-1009), hace una breve pausa (línea 1010), que contribuye a recrear una escena en la que se detiene a consultar el aparato, $y$, finalmente, reproduce el discurso escrito del mensaje en cuestión, en el que el chico le desea suerte (por los exámenes que va a realizar próximamente) y le envía un beso (líneas 1011-1012). En contraste con el sentimiento de tristeza que se expresaba inicialmente, ahora Eva se ríe para transmitir su alegría tras leer este mensaje y recibe la respuesta empática de Cris, que también se ríe y además hace una valoración positiva del chico (líneas 1013 1015). En los últimos turnos, la narradora-protagonista despliega sus emociones con especial intensidad, emitiendo interjecciones primarias o response cries (Goffman 1981), que simulan gritos o llantos de euforia y que provocan la risa de su amiga Cris (líneas 1016-1022).

En los turnos inmediatamente posteriores a los recogidos en este último extracto, Eva enfatiza su alegría y su satisfacción con esta relación que mantiene y expresa su deseo de que todo continúe así tras las vacaciones. Como ya hemos señalado, esta última historia es solo una muestra más del modo en que Eva enfoca sus experiencias dentro de esta relación, centrando la atención en los sentimientos, en la personalidad del chico, en los detalles de afecto que este tiene con ella y en el refuerzo progresivo de los vínculos y de la confianza entre ambos. Sus amigas responden ante sus expresiones de entusiasmo y euforia de forma empática y solidaria, transmitiendo alegría, pero también cierta cautela, recomendándole paciencia ante la incertidumbre que presenta la situación de cara al futuro. Como en historias analizadas previamente, en este último extracto también se aprecia una reproducción de los roles de género en el mundo de la historia, con la representación del personaje femenino en una actitud pasiva, esperando con ansia y cierta preocupación la llamada del chico.

\section{Discusión y Conclusiones Finales}

Los datos analizados en este artículo ofrecen un ejemplo de la realización de identidades de género en la conversación y de cómo esta categoría interactúa con la edad, el contexto comunicativo y la comunidad de práctica específica. Los extractos conversacionales que hemos examinado ponen de relieve el intercambio 
de confidencias sobre las relaciones con los hombres como un acontecimiento comunicativo que desempeña importantes funciones sociales e interpersonales entre las jóvenes participantes, actuando como un mecanismo de reafirmación de la amistad y de construcción de identidades juveniles que se presentan impregnadas de feminidad, teniendo en cuenta tanto el estilo de la interacción, como la "sustancia" o el contenido del discurso.

Desde una perspectiva general, la conversación que hemos abordado se basa en la producción de historias que tratan sobre personas y sobre las relaciones que se mantienen con ellas, ofreciendo múltiples detalles acerca de las mismas. Los estudios sobre género y narrativas conversacionales han destacado que esto es característico de las historias que cuentan las mujeres en la interacción con otras mujeres, en contraste con las historias de aventuras o grandes hazañas que suelen contar los hombres en los grupos masculinos (Johnstone 1990, 1993; Holmes 1997; Coates 2003). Además, las chicas comparten estas historias como una forma de "ponerse al día" acerca de sus vidas y desarrollan esta actividad durante casi treinta minutos, haciendo un trabajo intenso de retroalimentación a través de la emisión de respuestas mínimas, la aportación de opiniones sobre los hechos y la introducción de historias relacionadas con las cuestiones de debate que van surgiendo. En otros trabajos también se ha señalado que las historias de las mujeres tienen con frecuencia esta función de "ponerse al día" (Coates 2003, 2013) y que las señales de retroalimentación se hacen especialmente notables cuando las narrativas conversacionales se producen entre mujeres (Holmes 1997; Coates 2003).

De este modo se construye un estilo de comunicación estereotípicamente femenino, que se centra en la creación de intimidad, en la expresión de apoyo o solidaridad y en el refuerzo de la "conexión" entre las interlocutoras (Jones 1980; Johnson y Aries 1983; Tannen 1990, 1991; Coates 1996). Las participantes despliegan feminidad al recrear este estilo de interacción, pero también a través de la producción de historias que tratan más concretamente sobre las relaciones heterosexuales, abordándolas desde el punto de vista de los sentimientos y de la formación de pareja, como hemos destacado en el análisis. El discurso de las jóvenes reproduce valores que son propios de las generaciones más recientes, en la medida en que en él subyace una distinción entre "relaciones informales" y de "relaciones de noviazgo", que es característica de la gente joven (Megías y Ballesteros 2014). Sin embargo, aquí esas relaciones informales no son relatadas de acuerdo con los términos de libertad sexual y de falta de compromiso con los que suelen definirse, sino desde un punto de vista afectivo y como una etapa de transición hacia una relación de pareja.

Las historias que se cuentan están estrechamente relacionadas entre sí, en la medida en que giran en torno a los sentimientos, a los detalles de afecto o de falta del mismo por parte de los personajes masculinos, que aparecen representados de acuerdo con el estereotipo del hombre silencioso que no verbaliza sus sentimientos y del hombre independiente que aparenta falta de implicación con la relación de la que se habla. En contraste, las narradoras-protagonistas de estas historias despliegan sensibilidad, deseos de un mayor compromiso, alegría e incluso euforia ante las señales de reciprocidad afectiva que reciben o indignación ante la ausencia de las mismas. La realización de género también se produce dentro del mundo de las historias, con los personajes femeninos y masculinos actuando de acuerdo con los diferentes roles o características establecidos de forma convencional o estereotípica en el ámbito de las relaciones heterosexuales. 
Por tanto, la conversación está impregnada de género de múltiples formas. Las jóvenes no solo se identifican como mujeres heterosexuales, sino que además lo hacen ensalzando implícitamente determinados valores de la feminidad convencional o tradicional. Al mismo tiempo, no obstante, su discurso incluye elementos transgresores o innovadores, como el empleo de lenguaje ordinario o de expresiones malsonantes, que tradicionalmente han estado ligadas al habla de los hombres. Como ya señalamos en su momento, estos usos de lenguaje estereotípicamente masculino pueden interpretarse como una forma de marcar diferencias en comparación con las mujeres de generaciones anteriores y de reforzar así la construcción de una feminidad juvenil, en la que los tacos o las expresiones "fuertes" se aceptan y se utilizan como símbolo de igualdad con respecto a los hombres, al igual que las relaciones heterosexuales sin compromiso.

El intercambio de este tipo de confidencias, realizadas en la forma en que se ha mostrado, constituye una práctica común dentro de este grupo de amigas, con una función muy destacada en la realización de su amistad y de una versión de feminidad juvenil, que no es generalizable a otros grupos de jóvenes. Como explicábamos al revisar la trayectoria de los estudios sobre Lengua, Género y Sexualidad, la perspectiva constructivista/performativa pone el foco en los contextos comunicativos en los que se producen despliegues discursivos de género y en los mecanismos o recursos en los que estos se apoyan, evitando establecer diferencias estables entre los sexos, como se hacía previamente con la búsqueda de las "diferencias de género". En otros grupos de jóvenes, e incluso en otras conversaciones del mismo grupo de chicas, podrían emerger formas de feminidad juvenil diferentes a la que hemos identificado en este artículo (Acuña Ferreira en preparación) ${ }^{11}$.

El marco constructivista/performativo se defiende actualmente como un enfoque mucho más "dinámico" precisamente por esta razón, porque se parte de la base de que los individuos producen discursos de género que pueden variar en gran medida según el contexto comunicativo y que a menudo pueden resultar contradictorios entre sí. Estudios como el de Holmes (1997), por ejemplo, han destacado cómo, incluso dentro de una misma narrativa conversacional, producida por una hablante, pueden entrelazarse valores que son propios de una identidad femenina convencional y otros que construyen una identidad más asertiva y estereotípicamente poderosa, asociada con la masculinidad. Esta ambivalencia discursiva se interpreta como reflejo de la negociación constante que conlleva el género y la construcción de una identidad femenina o masculina, como una consecuencia del hecho de que "we are unwittingly involved in the ceaseless struggle to define gender", en palabras de Weedon (1987: 76; véase también Cameron 1995; Coates 2013).

No obstante, el modelo de feminidad juvenil que emerge en los datos aquí analizados parece reafirmar la coexistencia de valores igualitarios y tradicionales en el discurso de la gente joven que han apuntado los estudios sociológicos cualitativos revisados en Megías y Ballesteros (2014). Estos estudios, basados en grupos de discusión, observación participante o historias de vida, ponen de relieve un fuerte contraste entre la manifestación de actitudes igualitarias que defienden la libertad

11 Tampoco puede descartarse la producción de un discurso sentimental semejante en grupos de hombres, en cuyo caso se interpretarían como formas de desafío a los modelos predominantes de masculinidad o como despliegues de masculinidades alternativas (Coates 2003). 
sexual de las mujeres (en lo que se refiere a "relaciones informales", por ejemplo) y el mantenimiento de posturas clásicas en este terreno, como la consideración de que el papel activo en el cortejo debe asumirlo el hombre, la diferente forma en que se juzga la infidelidad masculina como algo "natural" y la femenina como una traición, el llamado doble estándar sexual o la gran importancia que dan las chicas a las relaciones de pareja y a los ideales románticos, a diferencia de los chicos. Ante estas discrepancias se ha señalado la necesidad de discernir hasta qué punto los discursos igualitarios identificados entre la gente joven indican una verdadera transformación de comportamientos y actitudes, o no son más que "un barniz formal que no altera las posturas profundas en las relaciones individuales" (Megías y Ballesteros 2014: 188).

Consideramos que el análisis de la conversación juvenil espontánea, de las prácticas comunicativas de la gente joven, puede contribuir a esclarecer esta cuestión. En nuestros datos conversacionales se ponen de manifiesto realizaciones de género en el ámbito de las relaciones heterosexuales que reafirman los roles y características tradicionales, así como una visión de las "relaciones informales" como una oportunidad de establecer una relación de pareja o una etapa de transición hacia la misma, que demuestra que la división entre ambos modelos de relaciones no está tan clara como se afirma en otros tipos de estudios. Por ello, pensamos que el estudio de las prácticas comunicativas de la gente joven en su entorno natural puede ofrecer claves muy valiosas para comprender mejor cómo funcionan las relaciones de género entre la gente joven y qué papel desempeña esta categoría social en la construcción de sus identidades. El concepto de acontecimiento comunicativo, en el que también se apoya el estudio de caso que hemos presentado, puede ser de gran utilidad como unidad de análisis en este tipo de investigaciones, orientadas a profundizar en la cultura juvenil desde una perspectiva de género. Así, la formación de un corpus de conversaciones juveniles relativamente amplio será una de las bases de los proyectos de investigación que planeamos desarrollar en el futuro.

\section{Agradecimientos}

Me gustaría expresar mi agradecimiento a las personas que colaboraron en la fase de obtención de datos conversacionales en los que se basa este trabajo.

\section{OBRAS CITADAS}

Acuña Ferreira, Virginia. 2002-2003. "Gendered emotive displays in complaint discourse". Estudios de Sociolingüística 3.2. y 4.1: 139-172.

Acuña Ferreira, Virginia. 2004. "Complaint stories in male contexts: the power of emotions". Spanish in Context 1.2: 181-213.

Acuña Ferreira, Virginia. 2008. "Complaint stories revisited: The "masculine" performance of a "feminine" narrative genre in a conversation among Galician men". Sociolinguistic Studies 2.2: 185-220.

Acuña Ferreira, A. Virginia. 2009a. Género y discurso. Las mujeres y los hombres en la interacción conversacional. Múnich: Lincom.

Acuña Ferreira, A. Virginia. 2009b. "Review of: Gendered talk at work. Janet Holmes. 2006. Oxford: Blackwell. Sociolinguistic Studies 3.1: 115-121. 
Acuña Ferreira, A. Virginia. 2011. La realización de quejas en la conversación femenina y masculina. Múnich: Lincom.

Acuña Ferreira, A. Virginia. 2012. "The humorous display of transgressor femininities: "Sharing a laugh" in Spanish/Galician friendly talk among young women". Sociolinguistics Studies 6.2: 121-147.

Acuña Ferreira, A. Virginia (en preparación). "Conversación juvenil e identidades de género. Despliegues de feminidad heterosexual en grupos de jóvenes veinteañeras".

Aries, Elizabeth. 1996. Men and women in interaction. Reconsidering the differences. Oxford: Oxford University Press.

Baron, Bettina y Helga Kothoff. eds. 2002. Gender in interaction. Perspectives on femininity and masculinity in ethnography and discourse. Amsterdam: John Benjamins.

Benwell, Bethan y Elizabeth Stokoe. 2006. Discourse and identity. Edinburgh: Edinburgh University Press.

Berger, Peter L. y Thomas Luckmann. 1966. The social construction of reality. Garden City, NY: Anchor Books.

Berger, Peter L. y Thomas Luckmann. 1976. La construcción social de la realidad. Trad. Silvia Zuleta. $3^{\text {a }}$ ed. en español. Buenos Aires: Amorrortu.

Bergvall, Victoria, Janet Bing y Alice Freed. eds. 1996. Rethinking language and gender research: Theory and practice. Londres: Longman.

Bing, Janet y Victoria Bergvall. 1996. "The question of questions: Beyond binary thinking". Rethinking language and gender research: Theory and practice. Ed. Victoria Bergvall, Janet Bing y Alice Freed. Londres: Longman. 1-30.

Bublitz, Wolfram. 1988. Supportive fellow-speakers and cooperative conversations. Amsterdam/Philadelphia: John Benjamins.

Bucholtz, Mary, A.C. Liang y Laurel A. Sutton. eds. 1999. Reinventing identities. The gendered self in discourse. Nueva York: Oxford University Press.

Bucholtz, Mary. 1999. "Why be normal?: Language and identity practices in a community of nerd girls". Language in Society 28: 203-223.

Bucholtz, Mary. 2003. "Theories of discourse as theories of gender: Discourse analysis in language and gender studies". The handbook of language and gender. Ed. Janet Holmes y Miriam Meyerhoff. Oxford: Blackwell. 43-68.

Butler, Judith. 1990. Gender trouble: feminism and the subversion of identity. Nueva York: Routledge.

Butler, Judith. 2001. El género en disputa: el feminismo y la subversión de la identidad. Trad. Mónica Mosour y Laura Manríquez. $1^{\mathrm{a}}$ ed. en español. México: Paidós.

Cameron, Deborah. 1995. "Rethinking language and gender studies: Some issues for the 1990s". Language and gender. Interdisciplinary perspectives. Ed. Sara Mills. Londres: Longman. 31-44.

Cameron, Deborah. 1997. "Performing gender identity: Young men's talk and the construction of heterosexual masculinity". Language and masculinity. Ed. Sally Johnson y Ulrike Hanna Meinhof. Oxford: Blackwell. 47-64.

Coates, Jennifer. 1988. "Gossip revisited: Language in all-female groups". Women in their speech communities. New perspectives on language and sex. Ed. Jennifer Coates y Deborah Cameron. Londres: Longman. 94-122.

Coates, Jennifer. 1996. Women talk. Conversation between women friends. Oxford: Blackwell. Coates, Jennifer. 1997a. "The construction of a collaborative floor in women's friendly talk". Conversation. Cognitive, communicative and social perspectives. Ed. T. Givón. Amsterdam: John Benjamins. 55-89.

Coates, Jennifer. 1997b. "Women's friendship, women's talk". Gender and discourse. Ruth Wodak. Londres: Sage. 245-262.

Coates, Jennifer. 1997c. "One-at-a-time: The organization of men's talk". Language and mas- 
culinity. Ed. Sally Johnson y Ulrike Hanna Meinhof. Oxford: Blackwell. 107-129.

Coates, Jennifer. 2003. Men talk. Stories in the making of masculinities. Oxford: Blackwell.

Coates, Jennifer. 2013. Women, men and everyday talk. Londres: Palgrave Macmillan.

Crawford, Mary. 1995. Talking difference. On gender and language. Londres: Sage.

De Klerk, Vivian. 1997. "The role of expletives in the construction of masculinity". Language and masculinity. Ed. Sally Johnson y Ulrike Hanna Meinhof. Oxford: Blackwell. 144-158.

Eckert, Penelope y Sally McConnell-Ginet. 1992. "Think practically and look locally: Language and gender as community-based practice". Annual Review of Anthropology 21: 461-90.

Eckert, Penelope y Sally McConnell-Ginet. 1999. "New generalisations and explanations in language and gender research". Language in Society 28: 185-203.

Edelsky, Carole. 1981. "Who's Got the Floor?". Language in Society 10 (3): 383-421.

Fishman, Pamela M. 1980. "Conversational insecurity". Language: Social psychological perspectives. Ed. Howard Giles, William Peter Robinson y Philip M. Smith. Oxford: Pergamon. 127-132.

Fishman, Pamela M. 1983. "Interaction: The work women do". Language, gender and society. Ed. Barrie Throne, Cheris Kramarae y Nancy Henley. Rowley, MA: Newbury House. 89-101.

Foucault, Michael. 1972. The archaeology of knowledge and the discourse on language. Nueva York: Pantheon.

Goffman, Erving. 1981. Forms of talk. Filadelfia: University of Pennsylvania Press.

Günthner, Susanne. 1997. "Complaint stories. Constructing emotional reciprocity among women". Communicating gender in context. Ed. Helga Hotthoff y Ruth Wodak. Amsterdam: John Benjamins. 179-218.

Haakana, Markku. 2007. "Reported thought in complaint stories". Reporting talk. Reported speech in interaction. Ed. Elizabeth Holt y Rebecca Clift. Cambridge: Cambridge University Press. 150-178.

Hall, Kira y Mary Bucholtz. eds. 1995. Gender articulated. Language and the socially constructed self. Londres: Routledge.

Henley, Nancy y Cheris Kramarae. 1991. "Gender, power and miscommunication". "Miscommunication" and problematic talk. Ed. Nikolas Coupland, Howard Giles y John M. Wiemann. Newbury, CA: Sage. 18-43.

Holmes, Janet. 1997. "Story-telling in New Zealand women's and men's talk". Gender and discourse. Ed. Ruth Wodak. Londres: Sage. 263-293.

Holmes, Janet. 2006. Gendered talk at work. Constructing gender identity through workplace discourse. Oxford: Blackwell.

Holmes, Janet. 2007. "Social constructionism, postmodernism and feminist sociolinguistics". Gender and Language 1.1: 51-67.

Hughes, Susan E. 1992. Expletives of lower working-class women. Language in Society 21: 291-303.

Johnson, Sally y Ulrike Hanna Meinhof. eds. 1997. Language and masculinity. Oxford: Blackwell.

Johnson, Fern y Elizabeth Aries. 1983. "The talk of women friends". Women's Studies International Forum 6: 353-361.

Johnstone, Barbara. 1990. Stories, Community, and Place. Bloomington: Indiana University Press.

Johnstone, Barbara. 1993. "Community and contest: Midwestern men and women creating their worlds in conversational storytelling". Gender and conversational interaction. Ed. Deborah Tannen. Oxford: Oxford University Press. 62-80.

Jones, Deborah. 1980. "Gossip: Notes on women's oral culture". Women's Studies International Quarterly 3: 193-198. 
Kendall, Shari y Deborah Tannen. 2001. "Discourse and gender". The handbook of discourse analysis. Ed. Deborah Schiffrin, Deborah Tannen y Heidi E. Hamilton. Oxford: Blackwell. 548-567.

Labov, William y Joshua Waletzky. 1967. "Narrative analysis". Essays on the verbal and visual arts. Ed. Judith Helm. Seattle: University of Washington Press. 12-44.

Labov, William. 1972. Language in the inner city. Filadelfia: University of Pennsylvania Press.

Lakoff, Robin. 1975. Language and woman's place. Nueva York: Harper \& Row.

Lakoff, Robin. 2004 [1975]. Language and woman's place. Text and commentaries. Ed. Mary Bucholtz. Oxford: Oxford University Press.

Lave, Jean y Etienne Wenger. 1991. Situated learning: Legitimate peripheral participation. Cambridge: Cambridge University Press.

Livia, Anna y Kira Hall, K. eds. 1997. Queerly phrased: Language, gender and sexuality. Nueva York: Oxford University Press.

Maltz, Daniel N. y Ruth A. Borker. 1982. "A cultural approach to male-female miscommunication". Language and social identity. Ed. John J. Gumperz. Cambridge: Cambridge University Press. 196-216.

Maltz, Daniel N. y Ruth A. Borker. 1995. "Los problemas comunicativos entre hombres y mujeres desde una perspectiva cultural”. Trad. Amparo Tusón. Signos. Teoría y Práctica de la Educación 16: 18-30.

Megías, Ignacio y Juan Carlos Ballesteros (2014). Jóvenes y género. El estado de la cuestión. Madrid: Centro Reina Sofía sobre Adolescencia y Juventud.

Mills, Sara. 2003. Gender and politeness. Cambridge: Cambridge University Press.

Motschenbacher, Heiko. 2010. Language, gender and sexual identity. Poststructuralist perspectives. Amsterdam/Philadelphia: John Benjamins.

Nakamura, Momoko. 2014. Language, gender and ideology. A genealogy of Japanese women's language. Amsterdam/Philadelphia: John Benjamins.

Pichler, Pia. 2009. Talking young femininities. Londres: Palgrave Macmillan.

Pujolar, Joan. 2002. Gender, power and heteroglossia: A sociolinguistic study of youth culture. Berlín: Mouton de Gruyter.

Pujolar, Joan. 2003. "La construcció del gènere en la interacció informal cara a cara: aspectes teòrics i metodològics". Estudios de Sociolingüistica 4.2: 485-503.

Rodríguez Yáñez, Xoán Paulo y Håkan Casares Berg. 2002-2003. “The Corpus of Galician/ Spanish Bilingual Speech of the University of Vigo: Codes tagging and automatic annotation”. Estudios de Sociolingüistica 3.2 y 4.1: 359-382.

Sacks, Harvey. 1992. Lectures on conversation (Vols. I y II, ed. G. Jefferson). Oxford: Blackwell.

Sacks, Harvey, Emanuel Schegloff y Gail Jefferson. 1974. "A simplest systematics for the organization of turn-taking for conversation”. Language 50: 696-735.

Schegloff, Emanuel. 1997. "Whose text? Whose context?" Discourse and Society 8.2: 165-187.

Speer, Susan A. y Elizabeth Stokoe. eds. 2011a. Conversation and gender. Cambridge: Cambridge University Press.

Speer, Susan A. y Elizabeth Stokoe. 2011b. "An introduction to conversation and gender". Conversation and gender. Ed. Susan A. Speer y Elizabeth Stokoe. Cambridge: Cambridge University Press. 1-27.

Sunderland, Jane. 2006. Language and gender. An advanced resource book. Londres: Routledge.

Talbot, Mary. 2010. Language and gender. An introduction. $2^{\mathrm{a}}$ ed. Oxford \& Cambridge: Polity Press.

Tannen, Deborah. 1990. You just don't understand. Women and men in conversation. Nueva York: William Morrow.

Tannen, Deborah. 1991. Tú no me entiendes. ¿Por qué es tan difícil el diálogo hombre-mujer? 
Buenos Aires: Javier Vergara Editor.

Tannen, Deborah. 1994. Gender and discourse. New York/Oxford: Oxford University Press. Tannen, Deborah. 1996. Género y discurso. Trad. Marco Aurelio Galmarini. Barcelona: Paidós. Tannen, Deborah. 1998. The argument culture: stopping America's war of words. New York: Ballantine.

Thorne, Barrie y Nancy Henley. eds. 1975. Language and sex: Difference and dominance. Rowley, MA: Newbury House.

Trömel-Plötz, Senta. 1991. Selling the apolitical. Discourse \& Society 2: 489-502.

Vila, María Rosa. 1987. "La segunda persona gramatical en función no deíctica". Revista Española de Lingüística 17: 56-68.

West, Candace, Michelle Lazar y Cheris Kramarae. 1997. "Gender in discourse". Discourse as social interaction. Vol. 2. Ed. Teun A. van Dijk. Londres: Sage. 119-143.

West, Candace, Michelle Lazar y Cheris Kramarae. 2000. "El género en el discurso". El discurso como interacción social. Vol 2. Ed. Teun A. van Dijk. Barcelona: Gedisa. 179-212.

West, Candace y Don H. Zimmerman. 1987. "Doing gender". Gender and Society 1: 125-151. Weedon, Chris. 1987. Feminist practice and poststructuralist theory. Oxford: Blackwell.

Zimmerman, Don H. y Candace West. 1975. "Sex roles, interruptions and silences in conversation". Language and sex: Difference and dominance. Ed. Barrie Thorne y Nancy Henley. Rowley, MA: Newbury House. 105-129.

ANEXO: CONVENCIONES DE TRANSCRIPCIÓN

\begin{tabular}{|l|l|}
\hline Línea de transcripción & Unidad de entonación \\
\hline$\uparrow$ & Entonación final ascendente \\
\hline$\downarrow$ & Entonación final descendente \\
\hline$?$ & Entonación final más o menos sostenida \\
\hline.$\cdot$ & Entonación típicamente interrogativa \\
\hline$<0.5>$ & Micropausa (de menos de un segundo) \\
\hline jo:: & Pausa medida en número de segundos \\
\hline $\begin{array}{l}\text { A: la típica callada? }= \\
\text { B: }=\text { sí }\end{array}$ & Sonido alargado \\
\hline $\begin{array}{l}\text { A: }[\text { y se nota que:: ya] } \\
\text { B: }[\text { que me lo dicen todo }]\end{array}$ & Alternancia de voces sin pausa \\
\hline (pues a ver si:: $)$ & Solapamiento de voces \\
\hline xx $)$ & Transcripción dudosa \\
\hline he he he & Segmento ininteligible \\
\hline LETRAS MAYÚSCULAS & Risa \\
\hline\{\} & Volumen relativamente alto o fortis \\
\hline$\{[p]\}$ & Segmento al que afecta el fenómeno \\
\hline$<$ voz simulada $>$ & Volumen relativamente bajo o piano \\
\hline & Comentarios adicionales \\
\hline
\end{tabular}

